\title{
LA CONSIDERACIÓN DEL SUJETO DE INVESTIGACIÓN COMO PARTE ACTIVA EN LA EMPRESA FARMACÉUTICA
}

\author{
María de la Luz Casas Martínez*
}

\begin{abstract}
Resumen: Aunque desde hace treinta años se diseñan estrategias internacionales para la protección del sujeto de investigación, la consideración del paciente como parte activa en la empresa farmacéutica es minimizada en la práctica. Una de estas estrategias se encuentra en la Declaración de Helsinki VI, donde el laboratorio se compromete a continuar suministrando posprotocolo el medicamento y/o procedimiento que haya sido benéfico para el paciente participante en el estudio; en la práctica, sin embargo, predomina el incumplimiento de dicho compromiso.

La bioética, como disciplina dialógica, trata de acercar a los involucrados buscando lenguajes y puntos comunes para llegar a acuerdos. El presente trabajo expone los argumentos propios de las industrias farmacéuticas, del derecho mercantil y de la ética general.
\end{abstract}

Palabras clave: investigación clínica, aspectos éticos, derechos de los pacientes, investigación bioética, Declaración de Helsinki

\section{THE CONSIDERATION OF THE SUBJECT PARTICIPANT IN RESEARCH AS ACTIVE PART IN THE PHARMACEUTICAL BUSINESS}

\begin{abstract}
Although for thirty years researchers involved in biomedical investigation have designed international strategies for the protection of the subject of investigation, the consideration of the patient as an active subject in the pharmaceutical business in practice is minimized. One of these compromises in the patient's protection is contained in the Helsinki VI Declaration, in which the laboratory accepts to continue the supply of the medicament and/or method studied postprotocol, when this has been proved to be a benefit to the participant in the research, in practice, this is not fulfilled.

Bioethics, as a pro- dialog discipline, tries to approach the involved sectors looking for languages or common points of view in order to have agreements. That is why I, in the present work, used the pharmaceutical laboratory's own arguments, commercial laws and general ethics.
\end{abstract}

Key words: clinical research, ethical aspects, patient's rights, bioethics research, Helsinki Declaration.

\section{A CONSIDERAÇÃO DO SUJEITO DE PESQUISA COMO PARTE ATIVA NA EMPRESA FARMACÊUTICA}

Resumo: Embora já fazem 30 anos que se desenharam estratégias internacionais para a proteção do sujeito da pesquisa, a consideração do paciente como parte ativa na empresa farmacêutica é na prática ainda desconsidero. Uma destas estratégias se encontra na Declaração de Helsinque VI, onde o laboratório se compromete a continuar administrando após o encerramento da pesquisa, o medicamento e/ou procedimento que tenha sido benéfico para o paciente participante do estudo. Na prática, ocorre o descumprimento do dito compromisso.

A bioética, como disciplina dialógica, trata de abordar todos os elementos envolvidos, buscando linguagem e pontos comuns para chegar a possíveis acordos. O presente trabalho expõe os argumentos próprios das industrias farmacêuticas, o direito mercantil e a ética em geral.

Palavras chave: pesquisa clínica, aspectos éticos, direitos dos pacientes, pesquisa em bioética, Declaração de Helsinque

Profesora investigadora en la Escuela de Medicina. Universidad Panamericana, México

Correspondencia: mcasas@mx.up.mx 


\section{Introducción}

María R., de 42 años, secretaria, madre de cuatro menores de edad. Desde hace tres años presenta diagnóstico de artritis reumatoide deformante, resistente a los tratamientos convencionales. Se encuentra con incapacidad desde hace tres meses. Acepta participar en un protocolo fase III de un nuevo AINES (PU 22036). A los quince días de tratamiento, la paciente presenta importante disminución sintomatológica y puede incorporarse nuevamente a su trabajo. Durante los seis meses de protocolo su mejoría es evidente. Al finalizar éste se le suspende el medicamento en estudio y se le prescribe terapia convencional. Un mes después solicita incapacidad laboral.

Las declaraciones de Helsinki VI ${ }^{1}$, la del Nuffield Council ${ }^{2}$ y las normas dictadas por el Council for International Organizations of Medical Sciences (CIOMS) (1) contienen algunas de las pautas éticas internacionales más importantes de la investigación biomédica. El compromiso en México, especialmente con la de Helsinki, es evidente, ya que aparece en forma obligada en las cartas de consentimiento bajo información en los protocolos de investigación en humanos.

El artículo 30 de dicha declaración garantiza a los pacientes que ingresan a un protocolo de investigación la continuidad del tratamiento una vez finalizado el protocolo, y cita así:

“30. Al final de la investigación, todos los pacientes que participan en el estudio deben tener la certeza de que contarán con los mejores métodos preventivos, diagnósticos y terapéuticos disponibles, identificados por el estudio."

World Medical Association. Helsinki Declaration. Available at http://www.wma.net/e/

Nuffield Council. Nuffield guidelines. Available at http:// www.nuffieldfoundation.org/

\section{Exposición del problema}

En la práctica, existe un incumplimiento de esta cláusula. Ello responde, a mi juicio, a un problema de justicia, puesto que el derecho a la atención de la salud es una garantía socio-jurídica avalada por la ética ${ }^{3} y$, además, porque el comportamiento humano requiere del soporte de la confianza para conservar los pactos.

En concreto, los problemas para la escasa aplicación del artículo se refieren al papel que se le otorga al paciente por los otros involucrados en la investigación y al temor de que su cumplimiento afecte el avance de las investigaciones en general. Trataré de analizar ambos problemas.

Sobre el papel del sujeto de investigación surge la pregunta: ¿quién debe recibir los beneficios de la investigación y sufrir sus cargas? El principio de justicia señala que ocurre una injusticia cuando algún beneficio al que una persona tiene derecho le es negado sin una buena razón para ello, o cuando alguna carga se impone indebidamente. Si esto es así, ¿tiene el paciente derecho a recibir beneficios después de su participación en un protocolo de investigación si éste fue evidentemente exitoso para él y para la empresa?

$\mathrm{Al}$ respecto, Greg Koski ${ }^{4}$, director de la Oficina de Protección de Investigación en Humanos de los Servicios de Salud de Estados Unidos, durante una conferencia en marzo de 2001, realizó un análisis crítico del artículo 30 y señaló que la medida, en muchos casos, no podía ser aplicada, ya que la efectividad de un producto variaba de una región a otra y que, por tanto, sería muy poco científico identificar como el "mejor tratamiento" los resultados de un solo evento de investigación.

\footnotetext{
World Health Organization. Alma-Ata Declaration. Available at www.who.int/hpr/NPH/docs/declaration_almaata.pdf Koski Greg. The limits of the intervention. Interview. http:/ /www.ncehr-cnerh.org/downloads/March\%202001/ KoskiNWmar01.PDF
} 
Alegó que tendrían que ser considerados también algunos hechos desde la perspectiva legal, pues el patrocinador podría entrar en conflicto con las leyes locales, proveyendo un producto que no estaba aprobado localmente para su uso o que había sido comercializado por otra organización.

Estas objeciones son fácilmente objetables. Respecto de la afirmación de que no puede avalarse con un solo protocolo el "mejor tratamiento", la referencia del artículo se encuentra en el caso particular, no en un sentido general. En el caso concreto la pregunta sería: para este paciente en particular, ¿ha sido más benéfico el medicamento y/o procedimiento utilizado experimentalmente?

Las otras objeciones -la variabilidad del producto por regiones y la aprobación de su uso en humanos- tampoco son válidas, porque ambos fueron factores considerados en forma previa a la aplicación del protocolo; si no hubiera sido así, no se habría podido realizar el estudio.

Respecto de la no aprobación local para su uso, la utilización del producto posprotocolo puede ser aceptada por el comité de bioética intrahospitalario como parte de una investigación especial fase IV $\mathrm{y} / \mathrm{u}$ otorgar un permiso provisional, ya que fue aceptado para uso en humanos. Es verdad que no se tiene permiso de comercialización pero, realmente, ese no es el punto, porque sí se tiene de utilización.

En estos términos, la solución de los problemas que se relacionan con la implementación de la cláusula no parece difícil. Lo que sí constituye un problema es el marco referencial en que se encuadra al paciente participante en investigación con fines comerciales. Las actitudes de los médicos y las empresas farmacéuticas varían desde las paternalistas a las pragmáticas, pero pocas veces a la consideración igualitaria de la participación del sujeto en todo el desarrollo del producto, incluyendo el comercial.
Creo que es necesario propiciar un cambio en la visión que se tiene del paciente participante en un protocolo y visualizar que su participación no concluye con el tiempo de aplicación del mismo. Para probar el punto, y ya que el lenguaje empresarial es específico, creo de utilidad analizar el papel del sujeto de investigación bajo este parámetro.

\section{Discusión}

Para la comprensión de la propuesta, es necesario partir de algunos argumentos, uno de ellos hace referencia a la disposición sobre el cuerpo humano. Desde la perspectiva kantiana(2), vigente en la mayoría de los países democráticos occidentales, el cuerpo humano no es comerciable; por lo tanto, esta propuesta no incluye una retribución económica a la participación del paciente posprotocolo, sino solamente la reevaluación de un derecho ya concedido pero poco comprendido por los integrantes del proceso de investigación biomédica.

Un supuesto a considerar, desde la perspectiva empresarial, es la participación en riesgos y beneficios por parte de los involucrados. El sujeto de investigación cubre ese supuesto: es un sujeto activo, con beneficios y riesgos durante su participación en el protocolo.

Una reciente investigación publicada por The Lancet(3) pone en duda la idea, ampliamente extendida, de que los pacientes oncológicos tienen más probabilidades de curación si son incluidos en ensayos clínicos que evalúen nuevos tratamientos. Para los autores del artículo no existe suficiente evidencia que apoye esa creencia, basándose en el análisis de 24 investigaciones publicadas al respecto. Sólo en tres de ellas se puso de manifiesto un beneficio evidente para los participantes respecto de los no participantes. Los pacientes, por tanto, son, como cualquier participante en una empresa, sujetos de riesgos y beneficios. 


\section{Modelo de asociación por participación}

Analizando los diferentes tipos de asociación mercantil, el modelo de asociación de participación(4) podría ser el más adecuado para hacer comprensible de mejor manera el papel del sujeto de investigación en la empresa farmacéutica.

Se debe dejar en claro que se trata de un modelo mercantil, es decir, basado en el lucro. Considerando que el cuerpo humano se encuentra fuera del comercio, como se expuso anteriormente, esta analogía no busca obtener para el sujeto un beneficio económico, sino una forma ajustada a derecho de disponer del fármaco a cuyo desarrollo se contribuyó.

La asociación por participación es un contrato por el cual una persona concede a otra, que le aporta bienes o servicios, una participación en las utilidades y en las pérdidas de una negociación mercantil o de una o varias operaciones de comercio(5).

Las obligaciones de los socios consisten en aportar los medios necesarios para la realización de un fin común. Las aportaciones pueden ser de dos clases: aportaciones de industria, realizadas por los socios industriales, y aportaciones de capital, por los socios capitalistas. Siendo así, el socio industrial contrae la obligación de hacer o proporcionar; el socio capitalista, de aportar económicamente.

La asociación por participación es semejante a la realizada con el fin de comercializar un producto farmacéutico porque en ésta hay:

- Un fin común (el desarrollo de un fármaco o tecnología).

- Una aportación, tanto capitalista como industrial.

- Una intención de compartir ganancias y pérdidas (éxito o fracaso en la comercialización).
- Una forma de contrato entre participante y empresa/investigador, representado por la Carta de Consentimiento bajo Información.

- Obligaciones de las partes.

Las asociaciones por participación se centran en el reparto de ganancias y pérdidas, las cuales deben ser otorgadas de acuerdo con las normas del contrato. En el caso de la cláusula 30 de Helsinki VI, estos términos se encuentran incluidos en el "contrato" representado por la Carta de Consentimiento, en donde siempre se hace referencia al respeto de esta declaración.

En esta empresa los participantes tienen riesgos y beneficios durante la realización del protocolo:

- Empresa: completar una fase de investigación. Comercialización versus no comercialización. Ganancia económica/prestigio versus pérdida económica.

- Investigador: prestigio/económico

- Sujeto de investigación: promoción de salud versus efectos secundarios y adversos (daño a la salud).

Si el protocolo finaliza exitosamente, existen ventajas para:

- La empresa, en forma de ganancia económica y de prestigio, por diez o más años (dependiendo de la duración de la patente).

- El investigador, en forma de prestigio y ganancia económica por tiempo indeterminado (dependiendo del impacto de la investigación).

- La ciencia, a través de un avance en el conocimiento.

El sujeto de investigación no obtiene beneficios, ya que se le suspende el tratamiento y/o 
diagnóstico o método preventivo a que dio pauta la investigación. Evidentemente, el sujeto se encuentra en desventaja respecto de los demás participantes en este tipo de sociedad.

Si el protocolo no es exitoso:

- La empresa pierde en parte o totalmente su inversión.

- El investigador se encuentra más protegido de pérdida, puesto que la utilidad de una investigación científica es válida tanto si se encuentran datos a favor como en contra.

- Los conocimientos científicamente adquiridos siempre son de utilidad.

El sujeto de investigación puede encontrarse en diferentes posiciones, según haya respondido al fármaco; en todo caso, al descontinuarse el estudio, si tuvo beneficio lo perderá y si tuvo efectos colaterales o secundarios también participa en la pérdida.

\section{Análisis desde una perspectiva ética}

¿Por qué es importante que se comprenda la necesidad del cumplimiento de un pacto? Siguiendo una idea de Tristam Engelhardt(6), para justificar la legitimidad de una bioética secular (capaz de guiar a los extraños morales a colaborar) existen solamente cuatro caminos: la fuerza, la conversión, la sólida argumentación racional y el acuerdo. En el caso de la investigación biomédica, el modo más adecuado de colaboración es el acuerdo, el cual se pacta, siguiendo nuevamente a Engelhardt, a través del principio de "permiso".

Esta forma constituye para la sociedad un compromiso que debe ser respetado. La visión de Engelhardt supone una ética de mínimos y es bajo ese parámetro que se asegura la obligatoriedad del cumplimiento de los pactos. Si esta premisa parte de la ética de mínimos, ¿cual será entonces la postura de la ética de máximos?
En la ética general, el respeto a las decisiones pactadas contribuye a un ambiente de confianza y de aceptación de responsabilidad. En este caso, por tratarse de un problema de justicia, el cumplimiento del compromiso pactado avalaría el hecho de que el valor de la justicia representa reconocimiento del valor y dignidad del ser humano, y que ese compromiso es mayor que el de intereses particulares. Los pactos suponen confianza y esta es la base de la solidaridad social; por tanto, constituye un valor que debe ser promovido.

La eticidad y la ley nos señalan que en caso de que se viera la conveniencia de la reforma de un artículo, se pactara nuevamente; pero mientras el lineamiento se encuentre vigente deberá ser respetado.

\section{Desventajas del cumplimiento del pacto}

Cumplir con los compromisos éticos siempre ha sido un reto y una auténtica lucha para cada miembro de la sociedad; sin embargo, sin esta tensión no habría posibilidad de progreso de la humanidad. Entonces, hay que reconocer que el cumplimiento de un deber ético suscita problemas.

En este caso, es muy posible que, en la práctica, se produzcan casos de conflictos de intereses entre la comunidad científica y las empresas farmacéuticas, ya que cabe la posibilidad de que las últimas otorguen oportunidades sólo a aquellos investigadores o instituciones que no lesionen sus intereses. Al verse en desventaja, algunos investigadores podrían ceder en su compromiso ético.

El riesgo es real. Pero, como en todo problema humano, no existe sólo una alternativa. La ética apuesta a que es posible obtener un bien de un mal inevitable. En este caso, la alternativa sería el valor ético de la solidaridad. $\mathrm{Si}$ todos los investigadores (o instituciones) se 
unen para hacer valer los compromisos éticos, ofrecerían una fuerza irresistible que repercutiría en un logro aún mayor que el mero cumplimiento del artículo.

Para ello, es inapreciable el pronunciamiento de los comités de bioética y de ética de la investigación, y de todas las otras instancias responsables del problema.

\section{Propuesta}

Considerando estos elementos, y para hacer operativa la cláusula 30 de Helsinki VI, propongo que se establezca un contrato de sociedad momentánea, es decir, para un solo acto jurídico y con las siguientes características:

\begin{tabular}{|c|c|}
\hline & OBLIGACIONES \\
\hline LABORATORIO & $\begin{array}{l}\text { a. Proporcionar el método preventivo, diagnóstico o } \\
\text { terapéutico identificado por el estudio. } \\
\text { b. Suspender dicha prestación, con una carta o receta } \\
\text { médica, cuando el médico tratante no considere al } \\
\text { fármaco "la mejor elección", o cuando el } \\
\text { medicamento pueda ser adquirido en forma genérica. }\end{array}$ \\
\hline
\end{tabular}

MÉDICO

\section{DERECHOS}

Que el médico le informe oportunamente.

Solicitar información sobre la evolución y estado clínico del paciente.

Proporcionar sólo el medicamento, sin hacerse cargo de ningún otro costo que suponga la toma del medicamento.

Utilizar la información recabada en su beneficio y en otras fases de investigación (IV).

Proporcionar a su paciente los mejores métodos preventivos, diagnósticos y terapéuticos disponibles, identificados por el estudio. Cumplir el principio terapéutico y de beneficencia. Propiciar un acto de justicia, por el que él esta éticamente comprometido.

Adquirir conocimiento sobre esta alternativa. medicamento", procederá a la solicitud del mismo. b. El médico tratante solicitará al laboratorio la continuación del tratamiento a su paciente si el fármaco se encontró en fase III o IV de investigación. c. Mediante una receta médica o carta institucional, expondrá un resumen clínico de la evolución del paciente al laboratorio, solicitando un determinado tiempo de tratamiento, sujeto a la patología y condiciones de que trate la terapéutica.

d. Mediante receta médica, que indique un tiempo determinado por el seguimiento del caso en cada patología, se surtirá el medicamento al paciente en el laboratorio.

Avisar al laboratorio de la suspensión del tratamiento.

\section{SUJETO DE Solicitar al médico el ingreso a esta prestación,}

INVESTIGACIÓN basado en la Carta de Consentimiento bajo Información, firmada previamente a la realización del protocolo, en la que se incluya que el mismo se adhiere a la Declaración de Helsinki VI.

Respetar las indicaciones del médico respecto a dosis, horario y otras recomendaciones para el uso del medicamento.

Cumplir con las revisiones médicas a que sea convocado, ya sea por parte del médico tratante o por el laboratorio. El costo de los controles necesarios será cubierto por su sistema de salud o por el paciente, si no tiene ninguno.

Utilizar el fármaco solamente para su uso y no hacer ningún lucro del mismo.
Recibir, en forma gratuita y expedita, el fármaco en cuyo protocolo participó como sujeto de investigación. 


\section{Conclusión}

Para la bioética, los principios de justicia y beneficencia son marcos de compromiso que deben ser promovidos. Es por ello que deben realizarse esfuerzos de diálogo entre los involucrados, a fin de construir acuerdos. En el caso que he expuesto, se busca la promoción de valores personales y sociales.

Uno de los principios más importantes que deben mantener quienes aceptan un pacto es guardar la palabra dada. Si no respetamos este principio vital de convivencia -en cualquier pacto: en nuestra empresa, con nuestros colaboradores, socios, pacientes- estamos cometiendo un grave error: se perderá credibilidad y confianza y se establecerá una relación "a la defensiva".

Si las empresas estuvieran exentas de los requerimientos morales, los resultados serían desastrosos. La sociedad no puede comportarse de una manera exclusivamente pragmática sin arriesgar su propio bienestar y sustentabilidad. Para la empresa, la reputación juega un papel importante; los argumentos de confianza propician acciones de colaboración y credibilidad como empresa ética.

Según el Censo del Milenio sobre responsabilidad social corporativa de 1999, la con- ducta ética, además de los méritos económicos, se está integrando en la definición de lo que hoy se entiende por "empresa excelente". Cualquier organización productiva que aspire a ser líder debe, ciertamente, crear riqueza para sus accionistas, pero requiere hacerlo cada vez más de manera ética y responsable.

Se requiere un mayor compromiso en el aspecto moral y social de muchas empresas. Éstas pueden atenerse solamente a "cumplir sus deberes", adhiriéndose a la ley y teniendo un nivel de desempeño "aceptable"; o, en un nivel más alto de acción, practicar una política de reciprocidad. El nivel superior sería, sin embargo, el de las empresas que dan más que lo justo legalmente: se comprometen más con la ética que con la ley.

Si se partiera de la confianza, la investigación biomédica se vería beneficiada. Una forma de obtener credibilidad se encuentra, precisamente, en el cumplimiento de los pactos. Es necesaria la observancia de todos los artículos contenidos en las declaraciones de protección ética de la investigación biomédica. Investigadores, sujetos de investigación y empresa farmacéutica conforman un tipo especial de asociación por participación, todos son sujetos de derechos y obligaciones y todos también sujetos morales.

\section{Referencias}

1. Council for International Organizations of Medical Sciences. International Ethical Guidelines for Biomedical Research Involving Human Subjects. Geneva: CIOMS; 2002.

2. Kant I. Fundamentación de la metafisica de las costumbres. 15a ed. Madrid: Espasa Calpe; 2001: 101.

3. Peppercorn JM, Weeks JC, Cook EF, Joffe S. Comparison of outcomes in cancer patients treated within and outside clinical trials: conceptual framework and structured review. The Lancet 2004; 363(9405): 263-70.

4. Mantilla Molina R. Derecho Mercantil. 9a ed. México: Porrúa; 2003: 221.

5. Agenda Mercantil ISEF. Ley General de Sociedades Mercantiles. Cap. XIII Art. 252. $8^{\mathrm{a}}$ ed. México: ISEF; 2004: 41.

6. Engelhardt T. The foundations of bioethics. New York: Oxford University Press; 1996: 67. 